\title{
Using Infrared Module and Laser Ranging Module to Monitor Tread Pattern of Powered Wheelchair
}

\author{
Chien-Yu Lu, ${ }^{1}$ Rong-Chu Sung, ${ }^{1}$ Lian-Wang Lee, ${ }^{2}$ \\ Wei-Lun Huang, ${ }^{1}$ and Te-Jen $\mathrm{Su}^{3,4^{*}}$ \\ ${ }^{1}$ Department of Industrial Education and Technology, National Changhua University of Education, \\ Changhua City 500, Taiwan, ROC \\ ${ }^{2}$ Department of Mechanical Engineering, National Chung Hsing University, \\ No. 145, Xingda Road, South District, Taichung City 40227, Taiwan, ROC \\ ${ }^{3}$ Department of Electronic Engineering, National Kaohsiung University of Science and Technology, \\ Kaohsiung City 807618, Taiwan, ROC \\ ${ }^{4}$ Graduate Institute of Clinical Medicine, Kaohsiung Medical University, Kaohsiung City 80708, Taiwan, ROC
}

(Received December 30, 2020; accepted April 6, 2021)

Keywords: powered wheelchairs, tread pattern, infrared module, laser ranging module

The purpose of this study is to detect the tread pattern of powered wheelchairs using an infrared module, a laser rangefinder, and a Raspberry Pi. The data are monitored by a computer to achieve the function of real-time monitoring in the backend. This system uses the Raspberry Pi OS and Python for coding. The information fed back from the infrared module and laser rangefinder are stored in Raspberry $\mathrm{Pi}$, and then sent to the backend computer for real-time monitoring. The advantages of this system are that it allows the user to monitor the tread pattern of a powered wheelchair in real time, and when an abnormal situation has occurred, this system can inform the user to carry out maintenance on the tire in time, so that the user can be more at ease in use and the manufacturer can improve their after-sales service for consumers.

\section{Introduction}

In recent years, with the increasing quality of medical resources, the world has been gradually moving towards aging societies. According to the data of the National Development Council, Taiwan became an aging society in 1993 and an aged society in 2018. It is estimated that Taiwan will become a super-aged society in 2026. ${ }^{(1)}$ As of April 2020, the population over 65 years of age in Taiwan was $15.57 \%$ and the number of people with disabilities in 2019 was 1.18 million, of which 60,000 people have been granted subsidies for living assistive devices. There is a demand for such devices for both people with disabilities and elderly people. ${ }^{(2)}$

Although there are many powered wheelchairs and electric scooters available on the market, ${ }^{(3-7)}$ there is no follow-up monitoring after sales. Usually, people only contact the company to ask for support when the devices have broken down and there is a major problem, inconveniencing both the company and the customers. If maintenance can be done in advance, it

${ }^{*}$ Corresponding author: e-mail: sutj@nkust.edu.tw

https://doi.org/10.18494/SAM.2021.3248 
will effectively reduce the labor requirement and inconvenience. For the above reasons, it is very important to build a system that allows customers to know the current situation of their wheelchairs. Although powered wheelchairs use both foam-filled and pneumatic tires, foamfilled tires are the most common. A pneumatic tire is shown in Fig. 1. Pneumatic tires have greater shock resistance, skid resistance, and balance, but they are more expensive and may puncture. In contrast, foam-filled tires (Fig. 2) have poorer shock resistance, skid resistance, and balance, but they do not puncture and can be maintained more easily, which is more convenient for powered wheelchair users. ${ }^{(8-10)}$

The tread pattern on the tire improves the grip of the vehicle, but long-term use will wear down the tread pattern. The reduced thickness of the tread pattern will decrease the gripping power. When the tread pattern is worn, the tire will lose its grip and make the vehicle skid, thus endangering users. Although all vehicles are now equipped with tire gauges, users still have to check the tread patterns beforehand. Therefore, if users can be warned in advance by a detector, the danger due to worn tread will be lessened. As vehicles are now equipped with various auxiliary technologies, the addition of a detector can help analyze tire tread patterns and ensure the safety of the vehicle, so as to increase the driving safety of users. This study aims to inform wheelchair users by monitoring the tread pattern. The software, hardware, and applications used for tread pattern monitoring comprise Raspberry $\mathrm{Pi}$, a laser rangefinder, an infrared transceiver module, and Python program editing.

\section{System Architecture}

The core of this system involves Raspberry Pi and a Python program. Raspberry Pi is a Linux-based single-chip computer developed by the Raspberry Pi Foundation in the UK. The memory of Raspberry Pi ranges between $256 \mathrm{MB}$ and 4 GB. It mainly uses a TransFlash (TF) card or a Secure Digital (SD) card as the storage medium, which is equipped with a USB interface, a High Definition Multimedia Interface (HDMI) video output (supporting voice output), and a terminal output, and is built in with an Ethernet/WLAN/Bluetooth internet linkage; in addition, it can be used in multiple operating systems, as shown in Fig. 3. Raspberry

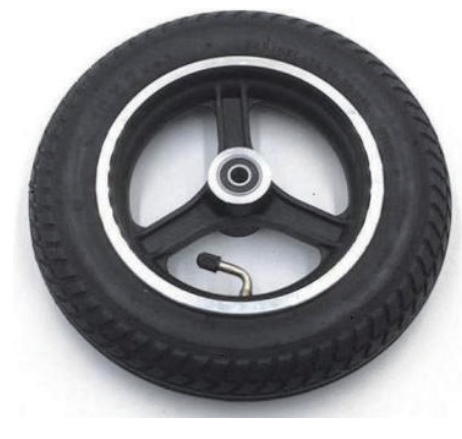

Fig. 1. (Color online) Pneumatic tire.

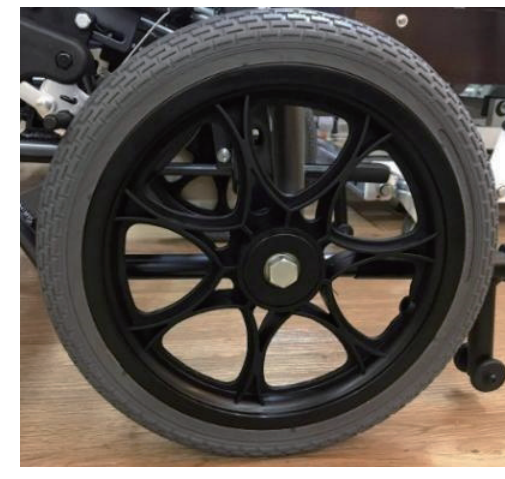

Fig. 2. (Color online) Foam-filled tire. 


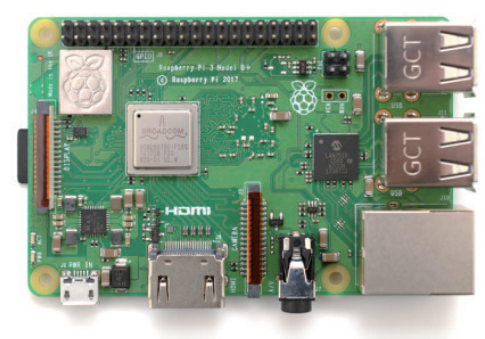

Fig. 3. (Color online) Raspberry Pi 3.

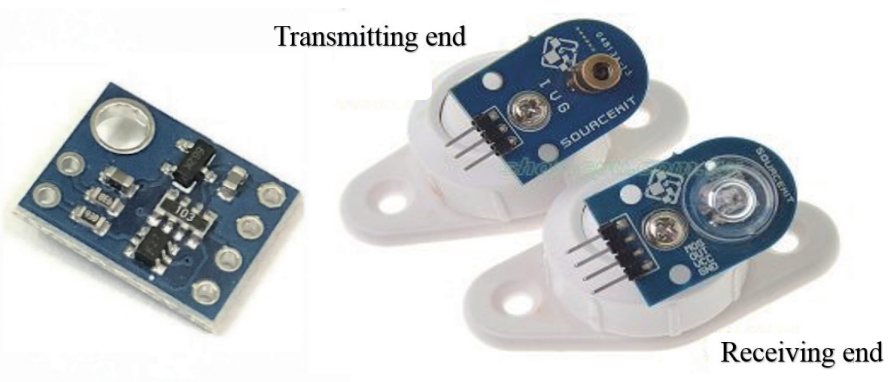

Fig. 4. (Color online) Laser rangefinder module.

Pi mainly uses the Linux core operating system. Currently, Raspbian is the official operating system of all Raspberry Pi models, while New Out Of Box Software (NOOBS) is the official operating system installation manager of Raspberry Pi. After installing NOOBS, the user can select whether to start up or install the operating system (OS). In addition to its own system, Raspberry Pi also supports other operating programs, such as the Windows 10 Core operating program released by Windows for Raspberry $\mathrm{Pi}^{(11)}$

For the sensor, a laser rangefinder and infrared module are used, as shown in Fig. 4. A GY530 VL53L0X laser rangefinder was adopted in this study, which has the smallest size on the market, a good filter, and an ultrahigh-speed photon detection array, so it can measure a long distance with high speed and accuracy. As we had to install the sensor in a small space and wanted its power supply to be supported by Raspberry Pi, this sensor was selected accordingly. ${ }^{(12)}$

The laser infrared module was paired up with the laser rangefinder. As Raspberry Pi does not have general-purpose input/output (GPIO) support, and the mainboard itself had no built-in analog input circuit, an additional analog-to-digital converter (ADC) had to be installed to convert the analog output value of these modules into digital information before it could be read by Raspberry Pi. Although there are many ADCs on the market, the differences among them mainly lie in the number of input pins and the conversion accuracy (bit). An MCP3008 ADC was used in this study. ${ }^{(13)}$

Python was used for the programming language in the program design. Python is a widely used interpreted, high-level, and general-purpose programming language. The design philosophy of Python emphasizes code readability and concise syntax. Compared with $\mathrm{C}++$ or Java, Python allows developers to express their ideas with fewer codes. Regardless of the size of the program, the language aims to make its structure clear. Python possesses a dynamic-type system and garbage collection function, which can automatically manage the memory usage and support multiple programming paradigms, including object-oriented, imperative, functional, and procedural programming. It has a huge and comprehensive standard library, and the Python interpreter can be executed in almost all operating systems, so it has good compatibility with other programs. ${ }^{(14)}$ The Atom editor was used in this study for editing Python, which is a text and program code editor developed by GitHub. Atom supports Linux, Windows, and macOS operating systems, as well as the plug-in written by Node.js. It has the built-in Git version control system provided by GitHub. Most of the derived suites have open-source licensing, and Atom 
can also be used as an integrated development environment. This editor is equipped with an intelligent AutoComplete function, which can make writing the program code faster and easier, and avoid possible errors. ${ }^{(15)}$ This section consists of three parts, which introduce the hardware architecture, the program architecture of the system, and the system synthesis. The hardware architecture of Raspberry Pi for receiving and processing data is introduced first, followed by the sensors used to detect tread patterns and the program architecture of the monitoring system. The structure of the tread pattern monitoring system composed of Raspberry Pi, a laser infrared contrast interrupter module, and a laser rangefinder module is shown in Fig. 5.

After writing a Python program with Atom, the edited program is entered into Raspberry Pi for program execution. The transmitting terminal of the laser infrared contrast interrupter module continuously sends signals. When the tread pattern height decreases to a set value due to wear, the receiving terminal will receive the signal and then send the data back to Raspberry Pi. The laser rangefinder module sends a signal to the tread pattern and sends the distance of the reflected signal to Raspberry Pi for timely monitoring. The real-time data are sent back to Raspberry Pi for analysis through the laser infrared contrast interrupter module and laser rangefinder module. After the analysis, the data are displayed on a mobile phone through Bluetooth transmission to achieve the monitoring function.

\section{(1) Hardware architecture}

The hardware of the tread pattern monitoring system chiefly comprises a processor and a sensor. First, the specifications of Raspberry Pi 3 and the ADC used are introduced.

Raspberry Pi has released Linux-based single-chip computers since 2015, from Raspberry Pi 1 to the latest Raspberry Pi. We adopted Raspberry Pi 3 released in 2018. As Raspberry Pi is equivalent to a small computer, its operating systems are easy to acquire, and there are many to choose from. In addition to the most common Raspberry Pi OS, options include Windows 10 Core, and Ubuntu Core. Despite its small size, light weight, and low power consumption, Raspberry Pi also has built-in Bluetooth and Wi-Fi functions. It is suitable for powered

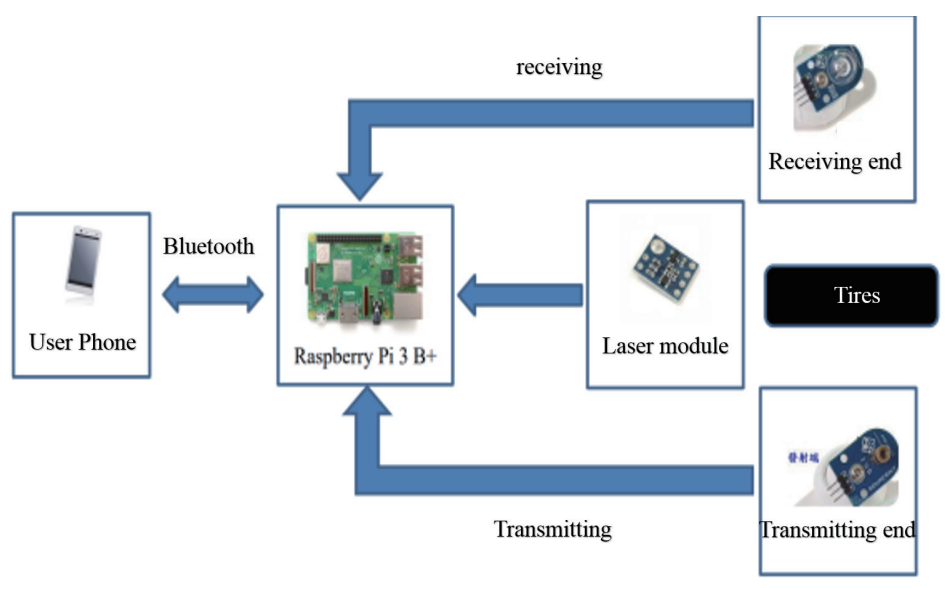

Fig. 5. (Color online) Architecture of tread pattern monitoring system 
wheelchairs that need to move around. Moreover, it has good expandability, making it an ideal choice in every aspect. However, as the laser infrared contrast interrupter module signal is an analog signal, which Raspberry Pi cannot read, an ADC is added at the receiving terminal of the laser infrared contrast interrupter module. Its function is to convert analog signals into digital signals, so that Raspberry Pi can read the signals sent back from the receiving terminal of the laser infrared contrast interrupter module.

There are many ADC models, but they only vary slightly, mainly in the number of input pins as well as conversion accuracy (bit). ${ }^{(13)}$ In this study, we chose the MCP3008 ADC to pair up with Raspberry Pi, with the ADC pin assignment shown in Fig. 6.

The sensor used in this study comprises a laser rangefinder module. The reason for choosing the module is that the space for installing the sensor is narrow. This sensor module is compact and lightweight and can use the power supply provided by Raspberry Pi. A GY-VL53L0X laser rangefinder module was chosen because it adopts the VL53L0X chip. This chip is provided with I2C interface communication, which is low in power consumption and has high accuracy over a range of $2 \mathrm{~m}$. It is the smallest model with reasonably high accuracy, making it very suitable for installation in a narrow space.

\section{(2) Software architecture}

Regarding the architecture and function of the main program of the tread pattern monitoring system, this system used the Atom editor to write the Python program, which was then executed by Raspberry Pi. The operation mode of the whole system is shown by the flow chart in Fig. 7. The whole system is divided into three major parts: sensor data acquisition, Raspberry Pi data identification, and Bluetooth notification.

\section{(3) System synthesis}

The data processing of the entire system is mainly organized by Raspberry Pi. First, the $\mathrm{ADC}, \mathrm{I} 2 \mathrm{C}$, and Bluetooth codes required in the system are entered into Raspberry $\mathrm{Pi}$, and the edited program is input into Raspberry Pi for execution. After execution, the sensor sends the data back to Raspberry Pi for recording, and Raspberry Pi then sends the data to users' mobile

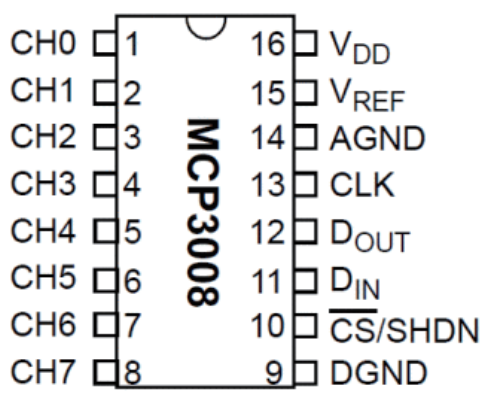

Fig. 6. MCP3008 pin assignment. ${ }^{(13)}$ 


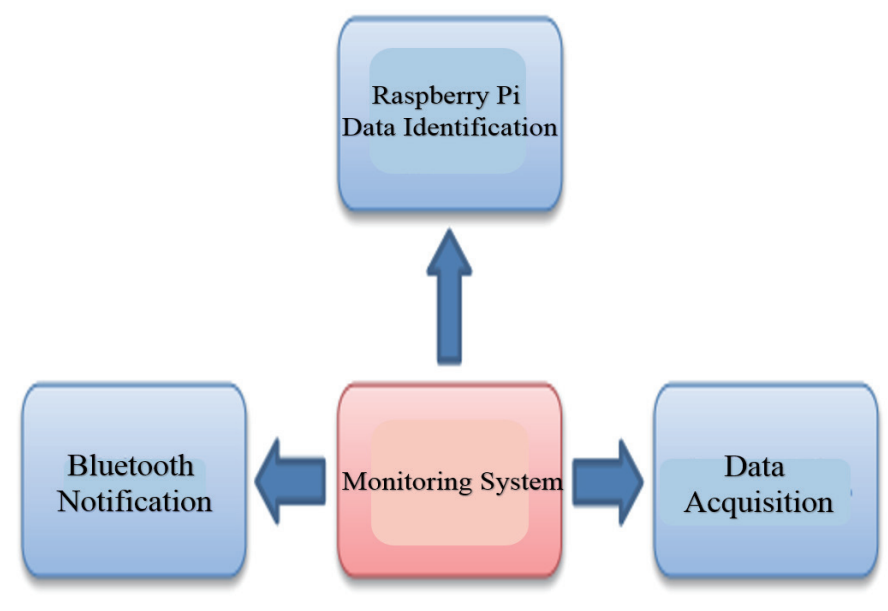

Fig. 7. (Color online) Tread pattern monitoring system architecture.

phones through Bluetooth, so that users know the current condition of the tread pattern in a timely manner. The operation procedure of the program is shown in the flow chart in Fig. 8.

\section{Experimental Analysis}

As Raspberry Pi cannot read analog signals, an ADC is needed to convert the signals. Raspberry Pi has to undergo a system test before adding any additional hardware. Some expansion modules produced by original manufacturers can be directly added to the system. However, the ADC is third-party hardware, so the corresponding codes have to be found on the Internet and added to Raspberry Pi before using Python for writing. The VL53L0X laser rangefinder module is often used in camera drones because of its compactness and light weight. However, it is a third-party module that requires the code to be driven by Raspberry Pi.

The logging module is a data storage function provided by Python. When writing programs, most of the time, the Print instruction is used directly to execute the action. However, the Print instruction cannot record the data obtained after the execution. If it is necessary to store the running data, the logging function must be used. Usually, when the Print instruction is used, the data obtained during the program running are displayed, but the logging module can classify the data into different levels. Although all the data are recorded during the storage, the data needed for display can be selected during the running. The data that need to be added or hidden can also be controlled in the code. For the logging module used in this study, the data displayed by the function are of the same color, while colored logs can change the color of the displayed data according to different functions so that users can read the data more quickly. ${ }^{(16)}$

The physical system is shown in Fig. 9. The power supply of the sensor is provided by Raspberry Pi, while the power supply of Raspberry Pi is provided by a PC USB connector in the simulation. The computer monitor is used for viewing the real-time data of Raspberry Pi, and the mobile app can be used for real-time monitoring through the Bluetooth connection with Raspberry Pi. 


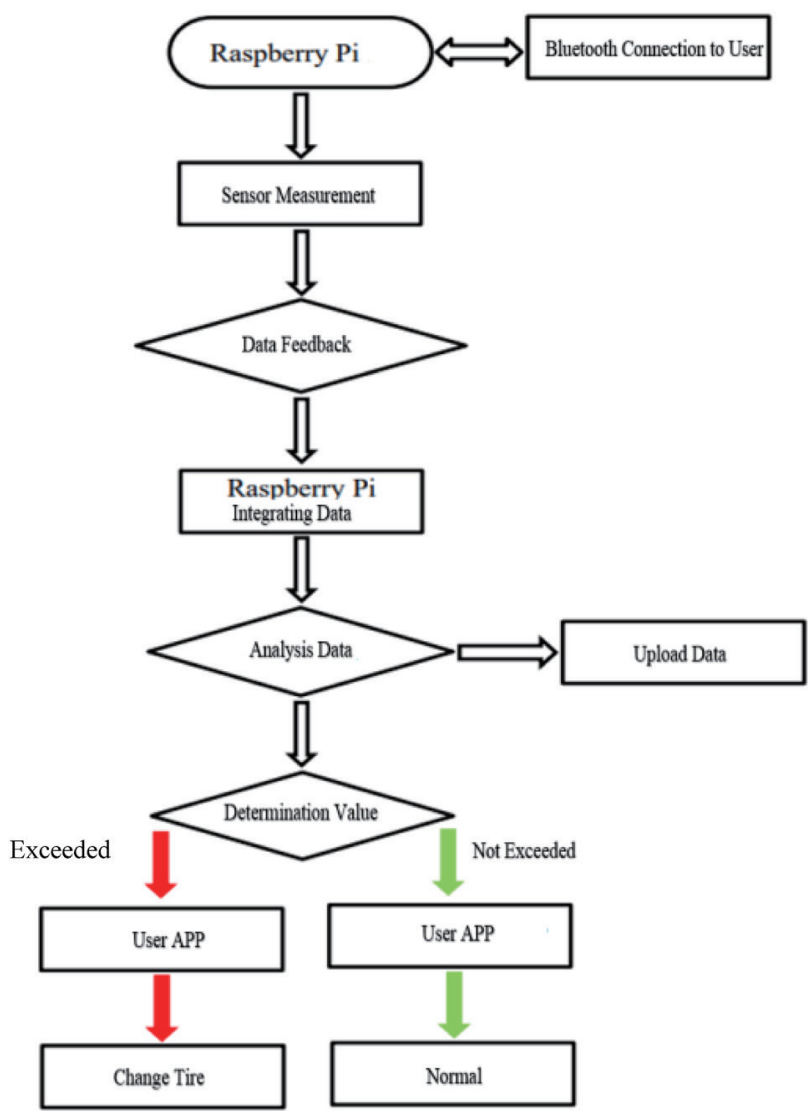

Fig. 8. (Color online) Operation flow chart of the tread pattern monitoring system.

The tread pattern monitoring mechanism is shown in Fig. 10. The red boxes are the laser infrared contrast interrupter modules, which are installed on both sides of the tire. The transmitting terminal is placed on the inner side of the tire and the receiving terminal is placed on the outer side. When the thickness of the tread pattern decreases, the infrared rays from the transmitting signal end are sent to the receiving end. When the receiving end receives the signal, the data will be sent back to the Raspberry Pi for analysis. The yellow box is the laser rangefinder module, which is placed directly above the tread pattern. The laser rangefinder module sends the laser onto the tread pattern to determine whether is too thin on the basis of the change of distance. Figure 11 shows the laser infrared contrast interrupter module and Fig. 12 shows the laser rangefinder module.

Before using the program, the functions to be enabled in Raspberry Pi have to be opened to make the program work normally. When using the laser infrared contrast interrupter module, laser rangefinder module, and ADC, the Serial Peripheral Interface (SPI) and I2C have to be opened. The SPI is used to control the ADC and the laser infrared contrast interrupter module, while I2C is used to control the laser rangefinder module. The instruction "sudo raspi-config" is entered in the command prompt of Raspberry Pi to access its "Advanced Options," as shown in Fig. 13. The option in line 5 is selected to open the SPI and I2C, as shown in Fig. 14. 


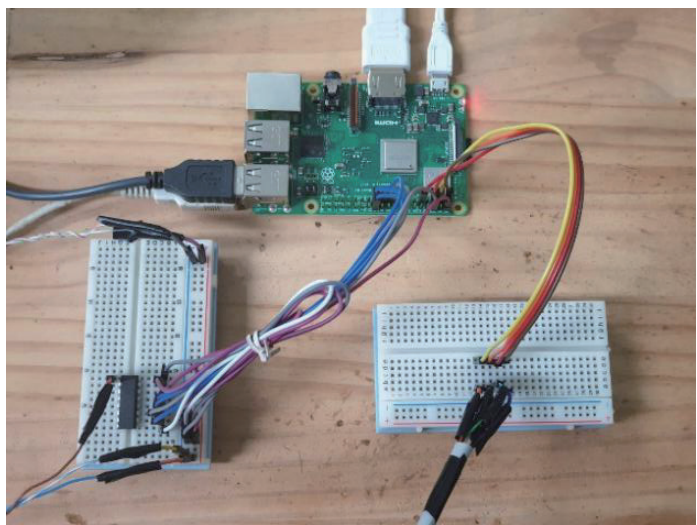

Fig. 9. (Color online) Tread pattern monitoring system.
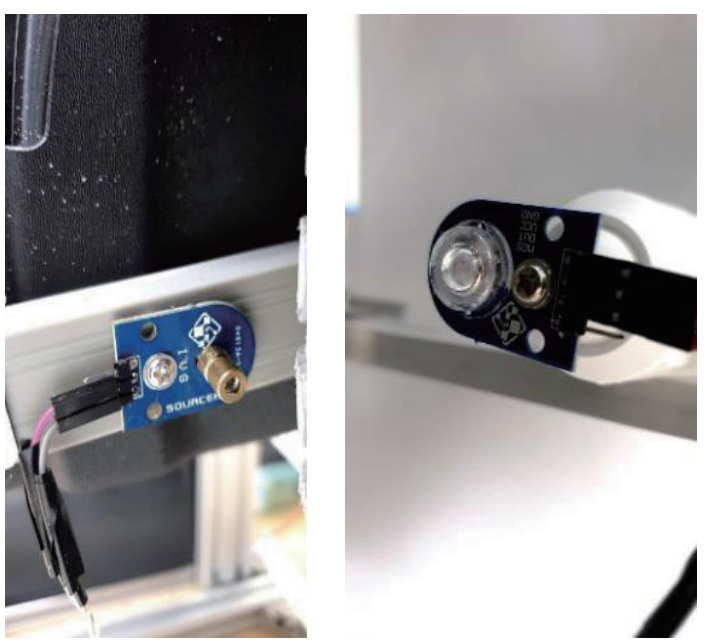

Fig. 11. (Color online) Laser infrared contrast interrupter module.

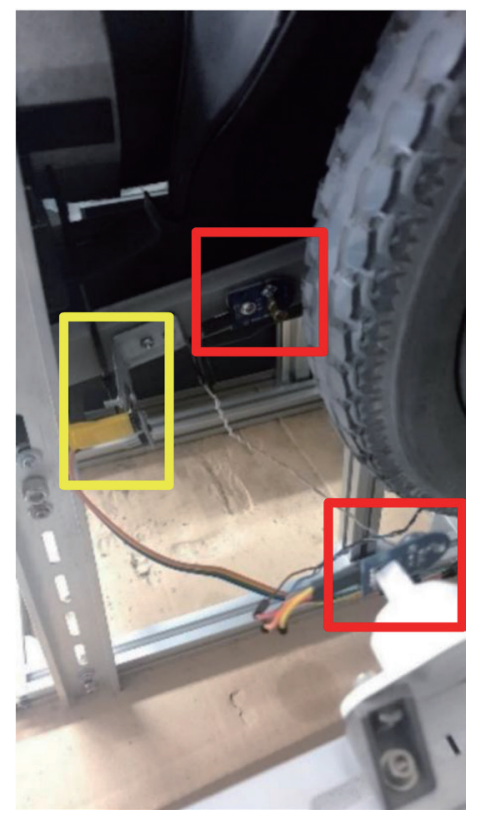

Fig. 10. (Color online) Tread pattern monitoring mechanism.

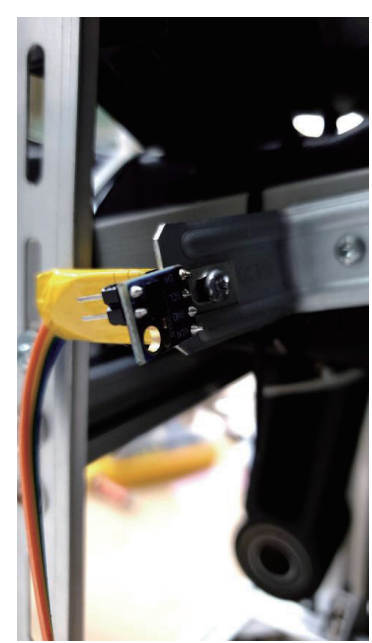

Fig. 12. (Color online) Laser rangefinder module.

The program is improved after startup. The program is saved into the set folder and the tread pattern monitoring system program is then retrieved from Raspberry Pi for running. The mobile phone and Raspberry Pi also require connection for pairing up before the connection in the mobile app. Only when the mobile phone symbol appears on the Bluetooth of Raspberry Pi can the pairing be considered successful. After successful connection, it is necessary to enter the mobile app for connection before the program control. Figure 15 shows the mobile app control interface. 


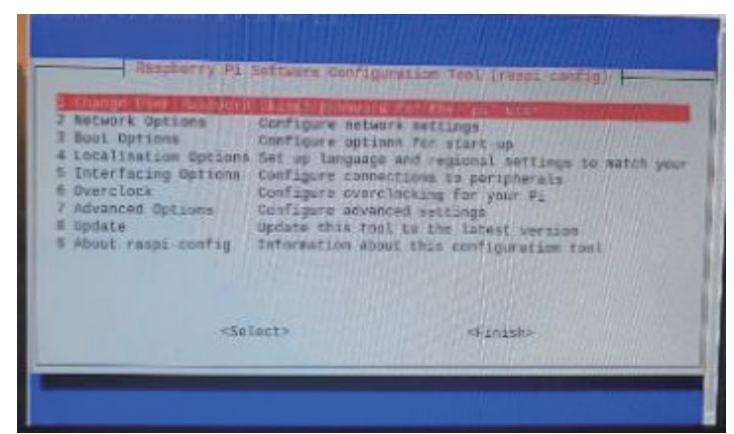

Fig. 13. (Color online) Raspberry Pi setting interface (Advanced Options).

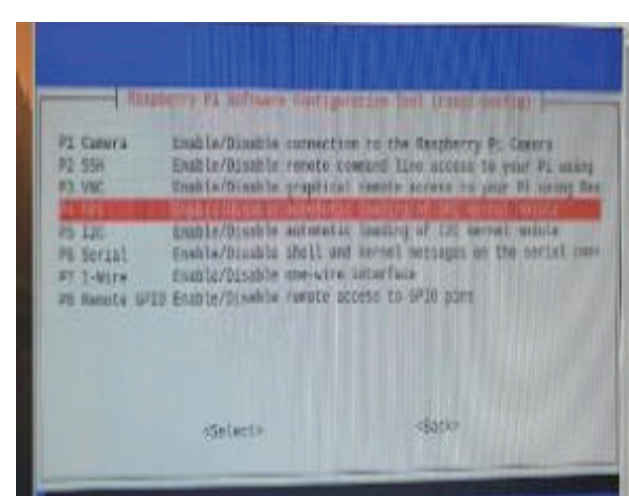

(a)

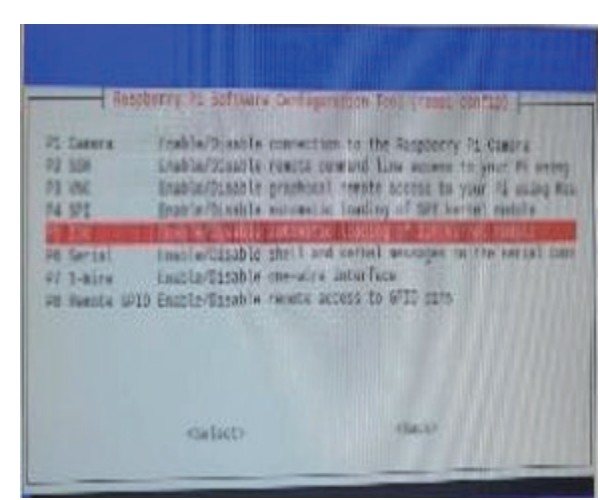

(b)

Fig. 14. (Color online) (a) SPI and (b) I2C (integrated circuit bus).

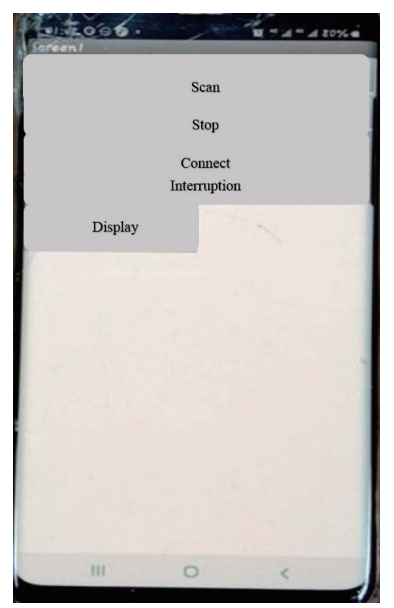

Fig. 15. (Color online) Mobile app control interface.

After Raspberry Pi pairs up successfully with the mobile Bluetooth, the mobile app screen entered is that shown in Fig. 16(a). When "Scan" is clicked on the current screen of the mobile phone, the location of the Raspberry Pi Bluetooth will appear. It is then necessary to click it and press the set connection button shown on the mobile app, and then press the display button 
shown on the mobile app. At this time, the screen will show whether the thickness of the tread pattern is normal according to analysis of the current sensor. When the tread pattern is normal, the display is that shown in Fig. 16(b); when it is abnormal, the display is that shown in Fig. 16(c).

The program data run in Raspberry Pi are divided into two groups: the data of the laser infrared contrast interrupter module and the data of the laser rangefinder module. The data of the laser infrared contrast interrupter module are shown in the red boxes of Figs. 17 and 18. Because the receiving terminal of the laser infrared contrast interrupter module produces errors owing to the influence of other light sources, to obtain accurate values, the program takes the average of every 50 potential signals of the receiver. The data shown in Figs. 17 and 18 are some of the 50 data. When the number of data reaches 50 , it will display whether the average value calculated is normal or abnormal. When it is normal, it means that the tread pattern has not worn excessively, as shown in Fig. 17. When the average value of the 50 data is zero, it means that the

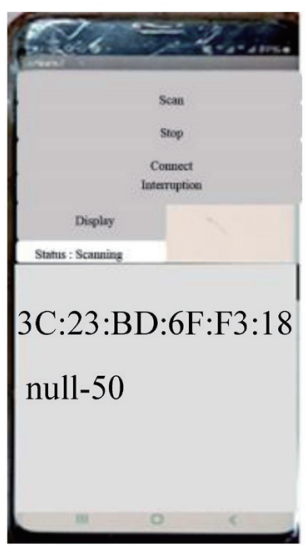

(a)

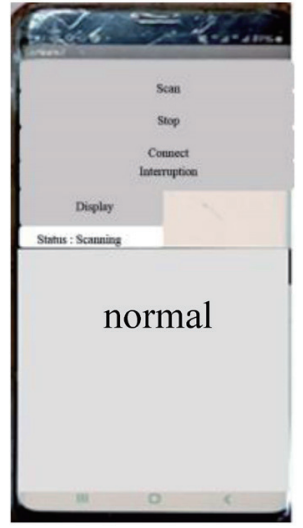

(b)

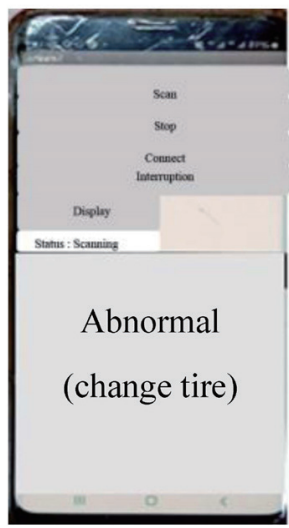

(c)

Fig. 16. (Color online) Screen on app showing (a) location of Raspberry Pi Bluetooth, (b) normal tread pattern, and (c) abnormal tread pattern.

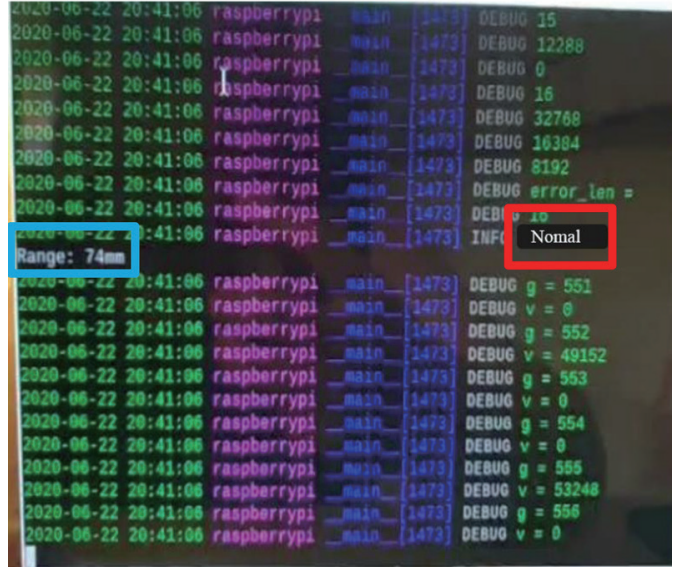

Fig. 17. (Color online) Normal data in Raspberry Pi.

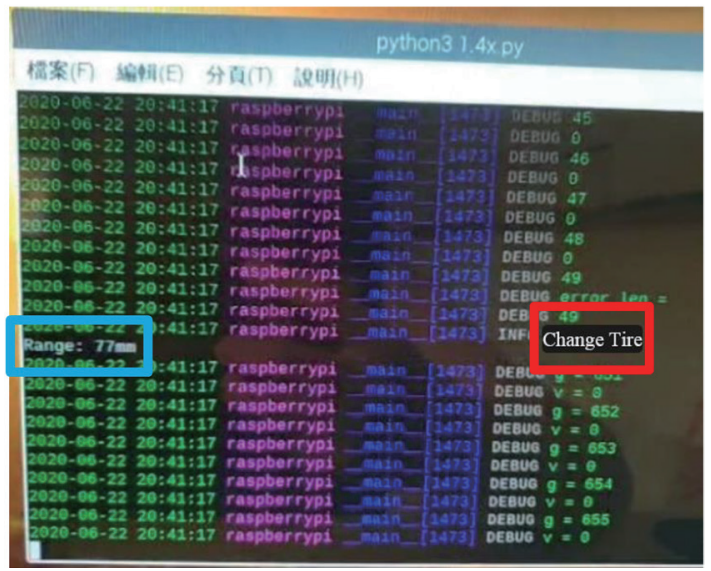

Fig. 18. (Color online) Abnormal data in Raspberry Pi. 
tread pattern has worn down and its thickness is inadequate; the system then displays this information, as shown in the red box of Fig. 18. In Figs. 17 and 18, the blue box is the data of the laser rangefinder module and the data of the laser infrared contrast interrupter module, which are displayed at the same time. The values of the laser rangefinder module will vary with the user and the user environment. To achieve an ideal value without considering the error, this system was evaluated on smooth ground. To allow manufacturers to read the data of the laser infrared contrast interrupter module and laser rangefinder module more easily and display the data in graphic forms, the laser rangefinder module and the laser infrared contrast interrupter module in the program are both displayed on the screen to provide the manufacturer with a basis for maintenance according to the data when users require maintenance. If the user's mobile phone and powered wheelchair have mobile networks or the user is in a place with a network connection, the data of the tread pattern depth (normal/abnormal) of the powered wheelchair can be transmitted to the mobile phone by means of network transmission, and the manufacturer can also monitor the data through the cloud server.

\section{Conclusion}

In this study, Raspberry Pi was applied to powered wheelchairs, allowing users to know the tread pattern depth of the wheelchair in a timely manner by means of Bluetooth transmission. This system can be applied to any powered wheelchair. As the laser infrared contrast interrupter module and laser rangefinder module used in this study are compact and lightweight and Raspberry Pi can store the data running in the program, the manufacturer can access the information in Raspberry Pi through the computer when the user requires maintenance, so that the manufacturer can select the appropriate tire materials in response to the needs of the user and obtain usage data from maintenance to facilitate subsequent services.

\section{References}

1 National Development Council: https://www.ndc.gov.tw/en/cp.aspx?n=2E5DCB04C64512CC (accessed June 2020).

2 Ministry of Interior Department of Statistics: https://www.moi.gov.tw/english/cl.aspx?n=7872 (accessed June 2020).

3 Karma Medical: https://www.karmamedical.com/ (accessed June 2020).

4 A. T. Hsieh: Research and Design of Electric Wheelchair, Master's Thesis (Department of Mechanical Engineering, Southern Taiwan University of Science and Technology, Taiwan, R. O. C., 2015).

5 Electric Wheelchair Control Interface: https://www.warmthings.com.tw/blog-post $/ \% \mathrm{E} 7 \% 89 \% \mathrm{~B} 9 \% \mathrm{E} 6 \% \mathrm{AE} \% 8$ A\%E4\%BB\%8B\%E9\%9D\%A2\%E9\%9B\%B (accessed June 2020).

6 M. A. Golovin and A. R. Sufelfa: 2019 IEEE Conf. Russian Young Researchers in Electrical and Electronic Engineering (EIConRus) 514-517.

7 B. F. Wu, Y. S. Chen, C. W. Huang, and P. J. Chang: IEEE Access 6 (2018) 28356. https://doi.org/10.1109/ ACCESS.2018.2839729

8 The History of Tire: https://zh.wikipedia.org/wiki/\%E8\%BC\%AA\%E8\%83\%8E\#\%E5\%AF\%A6\%E5\%BF\%83 $\% \mathrm{E} 8 \% 83 \% 8 \mathrm{E}$ (accessed June 2020).

9 The Difference between Foam Wheel and Inflatable Wheel: http://m.chaofen.cn/question/7402 (accessed June 2020).

10 T. F. Fwa, S. S. Kumar, K. Anupam, and G. P. Ong: J. Transport. Res. Board 12 (2009) 91. https://doi. org/10.3141/2094-10

11 Wikipedia: https://zh.wikipedia.org/wiki/\%E6\%A0\%91\%E8\%8E\%93\%E6\%B4\%BE (accessed June 2020). 
12 GY-530 VL53L0X: https://datasheet.lcsc.com/szlcsc/1909291034 Waveshare-VL53L0X-Distance-Sensor C431944.pdf (accessed June 2020).

13 MCP3008: https://chtseng.wordpress.com/2016/06/08/\%E6\%A8\%B9\%E8\%8E\%93\%E6\%B4\%BE\%E8\%AE\% 80\%E5\%8F\%96\%E9\%A1\%9E\%E6\%AF\%94\%E8\%B3\%87\%E8\%A8\%8A-mcp3008/ (accessed June 2020).

14 Wikipedia: https://zh.wikipedia.org/wiki/Python (accessed June 2020).

15 Atom editor: https://free.com.tw/GitHub-atom/ (accessed June 2020).

16 Coloredlogs: https://pypi.org/project/coloredlogs/ (accessed June 2020). 